\title{
Radiological and hemodynamic parameters in patients with suspected ventricular aneurysm and interventricular septal perforation after acute myocardial infarction: A comparison of non-invasive and invasive diagnostic modalities
}

\author{
JIA WU, MEIJUAN YAN, YUE CHEN, LONG CHEN and SHUANGFEI HU \\ Department of Anesthesiology, Zhejiang Province People's Hospital, \\ People's Hospital of Hangzhou Medical College, Hangzhou, Zhejiang 310014, P.R. China
}

Received October 6, 2019; Accepted January 22, 2020

DOI: $10.3892 /$ etm.2020.8754

\begin{abstract}
Transthoracic echocardiography is a rapid, sensitive and non-invasive technique for diagnosing ventricular septal perforation. Furthermore, left ventricular angiography is generally used for left ventricular aneurysm but right heart catheterization is the gold standard for septal perforation following myocardial infarction. The objectives of the present study were to compare radiological and hemodynamic diagnostic parameters of non-invasive methods with those of right heart catheterization in patients with suspected ventricular aneurysm and interventricular septal perforation after acute myocardial infarction. Data regarding demographics and clinical characteristics, as well as right heart catheterization, echocardiography and angiographic parameters of 199 patients examined within 21 days after myocardial infarction due to suspected ventricular septal defect indicated by persistent colic pain in the pre-cardiac region were collected and analyzed. Coronary angiography identified 149 (75\%) patients with single-vessel disease, 42 (21\%) patients with two-vessel disease and $8(4 \%)$ patients with triple-vessel disease. Transthoracic color Doppler echocardiography strengthened the diagnostic performance of right heart catheterization regarding segmental motor abnormalities but underestimated the right atrial pressure, systolic pulmonary artery pressure, mean pulmonary artery pressure and pulmonary capillary wedge pressure compared with right heart catheterization $(\mathrm{P}<0.0001$ for all).
\end{abstract}

Correspondence to: $\mathrm{Dr}$ Shuangfei $\mathrm{Hu}$, Department of Anesthesiology, Zhejiang Province People's Hospital, People's Hospital of Hangzhou Medical College, 158 Shangtang Road, Hangzhou, Zhejiang 310014, P.R. China

E-mail: floydMatthewsvzf@yahoo.com

\section{Abbreviation: TIMI, thrombolysis in myocardial infarction}

Key words: coronary angiography, left ventricular angiography, myocardial infarction, right heart catheterization, septal defect, transthoracic color Doppler echocardiography, ventricular aneurysm
Overall, there was no procedural complication requiring emergency intervention, no major complications and no conditions resulting in death due to diagnostic modalities. Transthoracic color Doppler echocardiography may strengthen the diagnostic performance of right heart catheterization regarding radiological measurements but underestimated hemodynamic measurements (level of evidence: 3 ).

\section{Introduction}

A true or pseudo ventricular aneurysm is the worst complication of transmural myocardial infarction (1). Left ventricular aneurysm after myocardial infarction is rare and usually occurs during the first week following the cardiac event but certain patients may present after a significant time following the cardiac event(s) (2). Interventricular septal perforation is also an infrequent but severe problem following myocardial infarction (3). Rapid diagnosis, immediate evaluation and management are required to prevent clinical destruction due to myocardial infarction (1). It is important to measure pulmonary hypertension in patients after myocardial infarction with possible septal defects (4). Several invasive and non-invasive imaging modalities are available to rule out this clinical entity.

Transthoracic echocardiography and right heart catheterization have been routinely performed in numerous medical centers worldwide. Right heart catheterization (floating pulmonary artery catheterization) is the 'gold standard' in the diagnosis of septal perforation but it is an invasive technique and generally performed when echocardiography is not accessible (4). The International Society for Heart \& Lung Transplantation guidelines (4) consider right heart catheterization as the most important diagnostic modality, as for critical patients with acute myocardial infarction and ventricular septal perforation, right heart catheterization is essential for evaluating pulmonary capillary wedge pressure, which cannot be accurately assessed by transthoracic echocardiography due to the septal perforation. Transthoracic echocardiography is a rapid, sensitive and non-invasive technique for diagnosing ventricular septal perforation (5) and may replace invasive pressure measurements. Transthoracic echocardiography may strengthen the diagnostic accuracy of right heart 


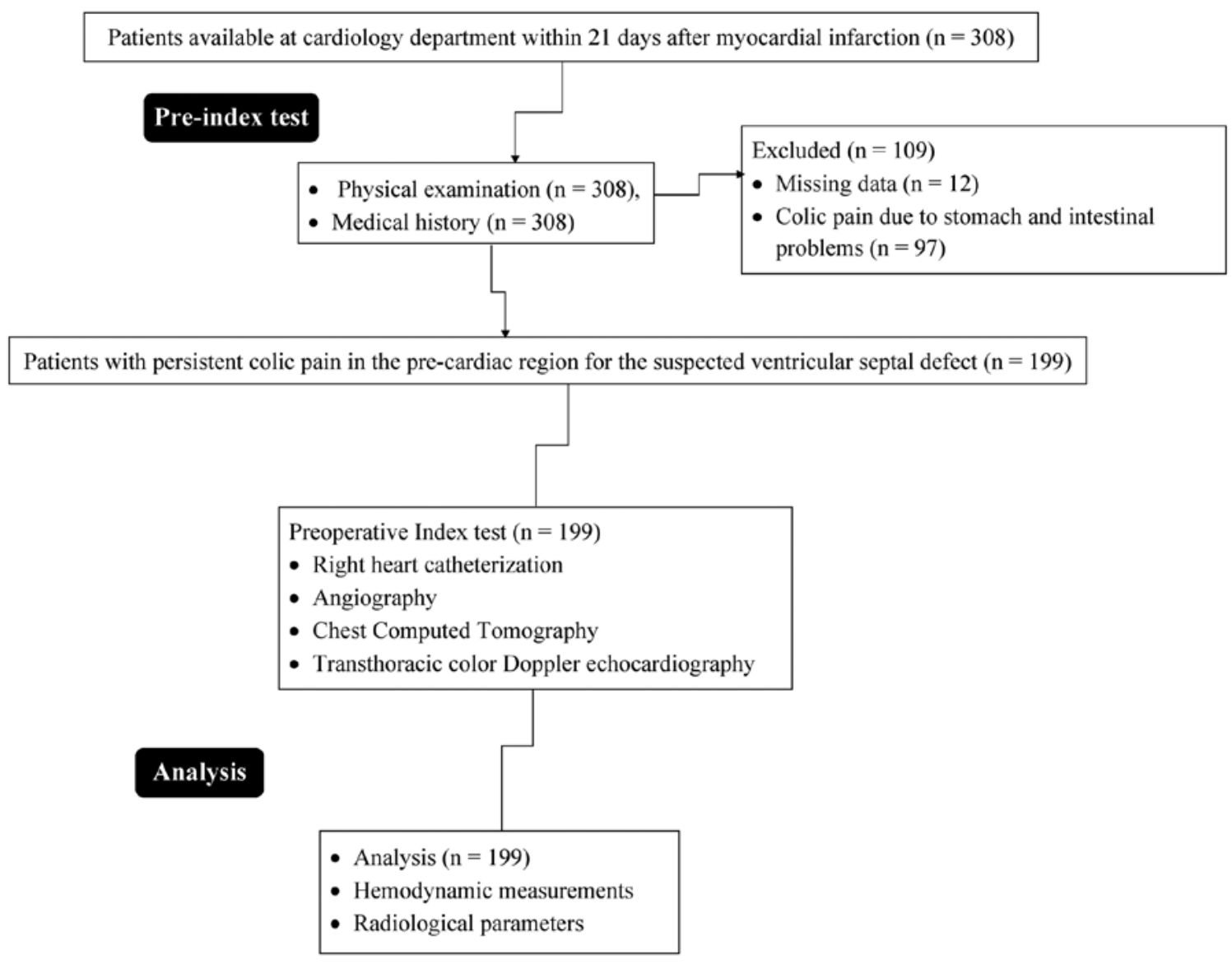

Figure 1. Flowchart of the analysis of patients in the present study.

catheterization (6). Left ventricular angiography is generally used for diagnosing left ventricular aneurysm (1) but also has a procedural risk for the patient (7).

The objective of the present study was to compare radiological and hemodynamic diagnostic parameters of non-invasive methods with right heart catheterization in patients with suspected ventricular aneurysm and interventricular septal perforation after acute myocardial infarction.

\section{Materials and methods}

Study population. From 16 February 2016 to 3 March 2019, a total of 308 patients (aged $\geq 18$ years) were admitted to the Department of Cardiology of Zhejiang Province People's Hospital, People's Hospital of Hangzhou Medical College (Hangzhou, China) and the referring hospitals within 21 days after myocardial infarction. Among them, 97 patients had no suspected ventricular aneurysm with interventricular septal perforation (colic pain only due to stomach and intestinal problems) and for another 12 patients, the complete data were not available. Therefore, data of these patients were excluded from the analysis. Data of demographic parameters, clinical characteristics, right heart catheterization parameters, as well as echocardiography and angiographic parameters of the remaining 199 patients with suspected ventricular septal defect due to persistent colic pain in the pre-cardiac region were included in the analysis. The flow diagram of the patients is presented in Fig. 1.
Data collection. Patient data regarding demographic and clinical characteristics, physical examination, right heart catheterization, echocardiography, chest CT, electrocardiogram, angiography and hemodynamic parameters were obtained from their medical records. The medical staff (the authors of the current study) of the institutes was involved in the collection of the data.

Right heart catheterization. Right heart catheterization was performed using proximal venous access via femoral, subclavian or internal jugular veins (8) by intensive care physicians (minimum of 3 years experience) of the institutes using a continuous pressure monitoring device (CARESCAPE ${ }^{\mathrm{TM}}$ V100 Monitor; GE Healthcare). Device selection, use of 'off-label' devices and multiple implantations were performed by the intensive care physicians themselves. The catheter was positioned in the pulmonary artery and the right heart cavity. The transducer was calibrated in the middle of the chest. The electrical and hemodynamic signals were digitally recorded. Pulmonary vascular resistance was recorded as per equation (Eq.) i (9). The cut-off values for pre- and post-capillary pulmonary hypertension were selected as $\geq 25$ and $\leq 15 \mathrm{mmHg}$, respectively (10).

Equations. The equations used were as follows: (i) Pulmonary vascular resistance=Mean pulmonary artery pressures-pulmonary capillary wedge pressure/Cardiac output; (ii) Right atrial pressure $=0.8+1.7$ (Early tricuspid Doppler inflow wave 
peak velocity/Doppler tissue imaging of early tricuspid annuli peak velocity); (iii) Systolic pulmonary artery pressure $=4 *($ Tricuspid regurgitation velocity $) / 2+$ Right arterial pressure; (iv) Mean pulmonary artery pressure $=(0.61 \mathrm{x}$ systolic pulmonary artery pressure) +2 ; (v) Cardiac output $=\Pi$ (Left ventricle outflow $\left.\operatorname{tract}^{2} / 4\right) *$ time integral of left ventricle outflow tract velocity*heart rate; (vi) Pulmonary capillary wedge pressure $=1.24$ (Early mitral Doppler inflow wave peak velocity/Doppler tissue imaging of early mitral annuli peak velocity)+1.9; (vii) Pulmonary vascular resistance=Systolic pulmonary artery pressure/Time integral of the right ventricular outflow tract velocity.

Angiography. All patients were subjected to cardiac catheterization for coronary angiography and left ventricular angiography (7) by cardiologists (minimum 3 years experience; blinded regarding results of right heart catheterization) of the institutes. The culprit vessels were evaluated as per thrombolysis in myocardial infarction (TIMI).

Transthoracic color Doppler echocardiography. Color Doppler transthoracic echocardiography was consecutively performed after right heart catheterization and angiography in all enrolled patients under the same conditions using an on-platform ultrasound system (MYLAB ${ }^{\mathrm{TM}}$ X7; Esaote S.p.A.) and $3-\mathrm{MHz}$ transducer at a speed of $100 \mathrm{~mm} / \mathrm{sec}$ (11) by sonographers (minimum 3 years experience; blinded regarding the results of angiography and right heart catheterization) of the institutes. The right atrial pressure was measured as per Eq. 2 (12). Systolic pulmonary artery pressure was calculated as per Eq. 3 (11). Mean pulmonary artery pressures were calculated as per Eq. 4 (9). Cardiac output was calculated as per Eq. 5 (11). Pulmonary capillary wedge pressure was calculated as per Eq. 6 (9). The pulmonary vascular resistance was calculated as per Eq. 7 (13).

Statistical analysis. The statistical analysis was performed using SPSS version 25 (IBM Corp.). The $\chi^{2}$ test of independence was used for comparison of categorical data and a Wilcoxon rank-sum test was used for continuous data. The confidence level of significance was set at $95 \%$ with $\mathrm{P}<0.05$ considered to indicate statistical significance.

\section{Results}

Demographic parameters and clinical characteristics. The mean age of the patients was $62.58 \pm 7.58$ years and the cohort included 77 (39\%) female patients. Patients presented with one or the other type of cardiovascular co-morbidity. All patients received standard medical treatment for acute myocardial infarction. Percutaneous coronary revascularization was performed in $121(61 \%)$ patients and was not performed in $78(39 \%)$ patients. The time of intra-aortic counterpulsation after myocardial infarction was $6 \pm 1$ days. The other demographic parameters and clinical conditions of the patients are presented in Table I.

Physical examination. The patients had a mean body mass index of $24.95 \pm 3.11 \mathrm{~kg} / \mathrm{m}^{2}$, no cyanosis, no jugular vein enlargement, no thyroid enlargement and no yellow stain on the skin sclera. The heart was not enlarged, the heart rhythm
Table I. Medical history of the patients enrolled $(n=199)$.

Characteristics Value

Systolic blood pressure ( $\mathrm{mmHg}$ )

$98.12 \pm 7.15$

Systolic pulmonary arterial pressure $(\mathrm{mmHg})$

$42.65 \pm 9.11$

Intra-aortic counterpulsation after myocardial

infarction (days)

Minimum

2

Maximum

20

Mean

$6 \pm 1$

Left ventricular ejection fraction (\%)

$45.12 \pm 8.47$

Site of acute myocardial infarction

Inferior

$97(48)$

Inferior-posterior

Anteroseptal

Surgical pre-procedural data

Surgical closure

Percutaneous closure

Co-morbidities

Diabetes

Smoking

Alcohol dependence

Hypercholesterolemia

$61(31)$

Mean

$2.51+0.15$

Cardiac function type ${ }^{\mathrm{a}}$

I

II

III

IV

Diabetic ketosis

Pulmonary infection

Present treatment $(\mathrm{s})^{\mathrm{b}}$

Aspirin

Atorvastatin

Frusemide

Spironolactone

$62(31)$

Bisoprolol

$72(36)$

Clopidogrel

$82(41)$

Percutaneous coronary revascularization

performed

Percutaneous coronary revascularization

$78(39)$ not performed

Categorical variables were expressed as $\mathrm{n}(\%)$ and continuous variables were expressed as the mean \pm standard deviation. ${ }^{a} \mathrm{New}$ York Heart Association classification: I, mild cardiac disease; II, mild to moderate disease; III, moderate disease; IV, severe cardiac condition.

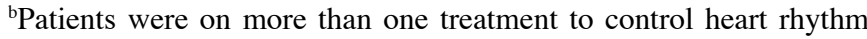
with/without oral hypoglycemic agent(s).

was uniform and the fourth intercostal space on the left edge had a systolic murmur. The lungs were clear, and no dry and wet voices were heard. The abdomen was soft and no tenderness or rebound tenderness was present. The liver and spleen 
Table II. Physical examination results in the cohort $(n=199)$.

\begin{tabular}{|c|c|}
\hline Characteristics & Results \\
\hline \multicolumn{2}{|l|}{ Age (years) } \\
\hline Range & $53-79$ \\
\hline Mean & $62.58 \pm 7.58$ \\
\hline \multicolumn{2}{|l|}{ Sex } \\
\hline Female & 77 (39) \\
\hline Male & $122(61)$ \\
\hline \multicolumn{2}{|l|}{ Ethnicity } \\
\hline Han Chinese & $186(93)$ \\
\hline Mongolian & $11(6)$ \\
\hline Tibetan & $2(1)$ \\
\hline Body mass index $\left(\mathrm{kg} / \mathrm{m}^{2}\right)$ & $24.95 \pm 3.11$ \\
\hline \multicolumn{2}{|l|}{ Adverse events } \\
\hline Nausea & $8(4)$ \\
\hline Vomiting & $2(1)$ \\
\hline Chill & $1(1)$ \\
\hline Fever & $2(1)$ \\
\hline Cough & $3(1)$ \\
\hline \multicolumn{2}{|l|}{ History of disease } \\
\hline Heart & $1(1)$ \\
\hline Liver (excludes hepatitis) & $1(1)$ \\
\hline Kidney & $2(1)$ \\
\hline Hepatitis & $2(1)$ \\
\hline Tuberculosis & $2(1)$ \\
\hline Thyroid & $2(1)$ \\
\hline Edema in both lower limbs & $2(1)$ \\
\hline
\end{tabular}

Categorical variables were expressed as $\mathrm{n}(\%)$ and continuous variables were expressed as the mean \pm standard deviation.

under the ribs had no tenderness. There were two patients with edema in both lower limbs The other results of the physical examination are presented in Table II.

Angiography. Coronary angiography identified 149 (75\%) patients with single-vessel disease, $42(21 \%)$ patients with two-vessel disease and $8(4 \%)$ patients with the triple-vessel disease (data not shown). The right coronary artery was blocked in $99(50 \%)$ patients, the left anterior descending artery was blocked in $83(42 \%)$ and the circumflex artery was blocked in 17 patients (3\%; Fig. 2). Left ventricular angiography revealed abnormal wall motion and ventricular aneurysm. None of the patients had intravenous spontaneous thrombolysis (Fig. 3).

Chest CT. In most of the patients, the chest CT revealed inflammation of the bilateral lungs, bilateral pleural effusion with segmental lung tissue insufficiency and pulmonary emphysema.

Transthoracic color Doppler echocardiography. Apical ventricular aneurysm formation with interventricular septum

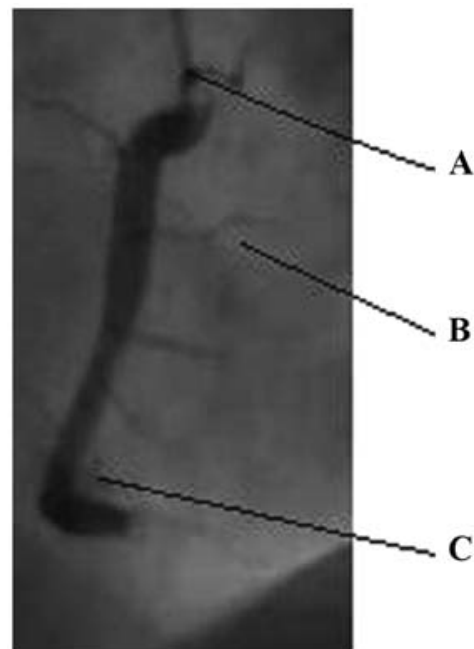

Figure 2. Coronary angiography image of a representative patient aged 70 years (body weight, $70 \mathrm{~kg}$; body height, $162 \mathrm{~cm}$; sex, male.) of the right coronary dominant type. The left major trunk exhibited no obvious stenosis. The anterior descending branch had $99 \%$ stenosis in the proximal segment and $70-80 \%$ stenosis in the middle segment with a TIMI blood flow grade 3 . The circumflex branch had $40-50 \%$ stenosis near the middle segment and a TIMI blood flow grade 3 . The right coronary artery had $40-50 \%$ stenosis in the proximal segment, $30-40 \%$ stenosis in the middle segment and $40 \%$ stenosis in the distal segment with a TIMI blood flow grade 3. A, coronary artery, B, ventricular margin in anterior descending branch, $\mathrm{C}$, septum in anterior descending branch. TIMI, thrombolysis in myocardial infarction.

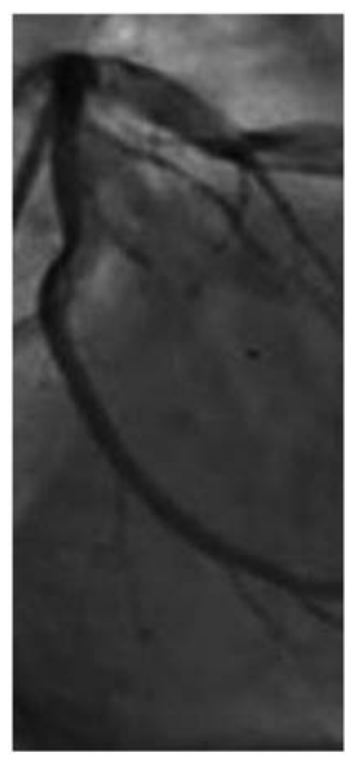

Figure 3. Left ventricular angiography image of the patient aged 70 years (body weight, $70 \mathrm{~kg}$; body height, $162 \mathrm{~cm}$; sex, male).

perforation, left ventricular wall segmental motor abnormalities (widening anterior wall motion weakened), aortic sclerosis, ascending aorta widening, mild mitral regurgitation, moderate tricuspid regurgitation, moderate pulmonary hypertension, left ventricular systolic and diastolic dysfunction were observed on transthoracic color Doppler echocardiography in all patients (see representative image in Fig. 4).

Cervical vascular ultrasound. Bilateral carotid atherosclerosis with multiple plaque formation, moderate left stenosis 
Table III. Hemodynamic measurements by right heart catheterization and transthoracic color Doppler echocardiography in the cohort $(n=199)$.

\begin{tabular}{lcrc}
\hline Hemodynamic characteristics & Right heart catheterization & Echocardiography & P-value \\
\hline Heart rate (beats per minute) & $74 \pm 14$ & $75 \pm 15$ & 0.492 \\
Oxygen saturation (\%) & $92 \pm 4$ & $91 \pm 5$ & 0.028 \\
Mean blood pressure (mmHg) & $96 \pm 2$ & $95 \pm 1$ & $< \pm 2$ \\
Right atrial pressure (mmHg) & $8 \pm 2$ & $59 \pm 3$ & $<0.0001$ \\
Systolic pulmonary artery pressure (mmHg) & $61 \pm 2$ & $34 \pm 3$ & $<0.0001$ \\
Mean pulmonary artery pressure (mmHg) & $39 \pm 2$ & $5.85 \pm 0.15$ & $<0.0001$ \\
Cardiac output (l/min) & $6.15 \pm 0.21$ & $11 \pm 2$ & $<0.0001$ \\
Pulmonary capillary wedge pressure (mmHg) & $14 \pm 1$ & $3.72 \pm 0.18$ & $<0.0001$ \\
Pulmonary vascular resistance (Wood units) & $4.85 \pm 0.25$ & $<0.0001$
\end{tabular}

Values are expressed as the mean \pm standard deviation. A Wilcoxon rank-sum test was used for statistical analysis.

Table IV. Procedural complications in the patients $(n=199)$.

\begin{tabular}{|c|c|c|c|c|}
\hline Hemodynamic characteristics & Right heart catheterization & Echocardiography & Angiography & P-value \\
\hline Carotid artery puncture & $5(3)$ & $0(0)$ & $0(0)$ & 0.007 \\
\hline Hematoma & $3(1)$ & $0(0)$ & $2(1)$ & 0.244 \\
\hline Coagulopathy & $2(1)$ & $0(0)$ & $0(0)$ & 0.134 \\
\hline Infections & $0(0)$ & $0(0)$ & $1(1)$ & 0.367 \\
\hline Respiratory insufficiency & $0(0)$ & $0(0)$ & $2(1)$ & 0.134 \\
\hline
\end{tabular}

Values are expressed as $\mathrm{n}(\%)$. The $\chi^{2}$ test of independence was used for statistical analysis.

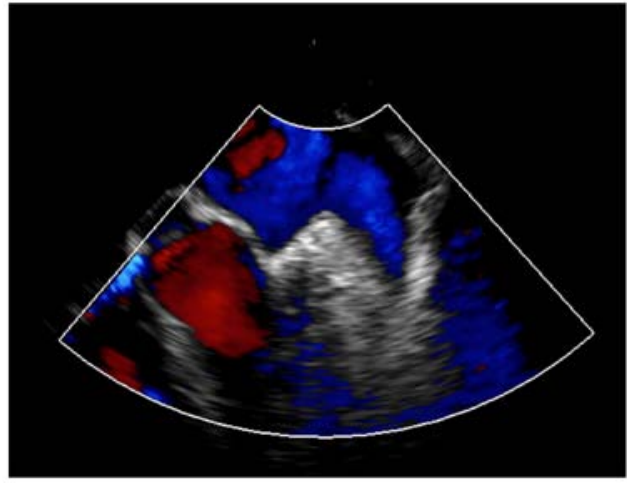

Figure 4. Transthoracic color Doppler echocardiogram of the patient aged 70 years (body weight, $70 \mathrm{~kg}$; body height, $162 \mathrm{~cm}$; sex, male) demonstrating mild mitral regurgitation.

of the left common carotid artery, right carotid sinus and internal carotid artery with severe or moderate stenosis were observed on cervical vascular ultrasound in certain patients (10 patients).

Electrocardiogram. The majority of patients had a sinus rhythm in line with acute extensive anterior wall and inferior myocardial infarction on the electrocardiogram. In addition, left chest lead, limb lead QRS complex low voltage and QTc interval prolongation were observed.
Hemodynamic measurements. All hemodynamic parameters exhibited significant differences between right heart catheterization and transthoracic color Doppler echocardiography $(\mathrm{P}<0.05$ for all) except heart rate $(\mathrm{P}=0.492)$. Transthoracic color Doppler echocardiography underestimated post-capillary pulmonary hypertension (Table III).

Procedural complications. Overall, there was no procedural complication requiring emergent intervention, major complications or any condition resulting in death due to diagnostic modalities. Echocardiography exhibited less complications than catheterization (and angiography; Table IV).

\section{Discussion}

Right heart catheterization and transthoracic color Doppler echocardiography may provide hemodynamic parameters for patients with ventricular septal defect following myocardial infarction. In the present study, coronary and left ventricular angiography, physical examinations, chest CT and electrocardiogram did not provide sufficient information for hemodynamic parameters. Central hemodynamic parameters are important for the diagnosis, classification (mean pulmonary artery pressure and pulmonary capillary wedge pressure) and prognosis (the right atrial pressure) of the ventricular aneurysm with interventricular septal perforation (9). The present study recommended either 
right heart catheterization or transthoracic color Doppler echocardiography as examinations to provide pre-operative indexes in patients with suspected ventricular aneurysm with interventricular septal perforation after acute myocardial infarction.

Angiography provides information on the degree of stenosis and culprit vessel(s). Cervical vascular ultrasound may also provide information on atherosclerosis. Three-dimensional imaging modalities may reveal acute mechanical complications of myocardial infarction (5). Chest CT is effective in detecting pseudo-aneurysms, abscesses and vegetation (14). Angiography, cervical vascular ultrasound and chest $\mathrm{CT}$ are an essential adjunct method to transthoracic color Doppler echocardiography or right heart catheterization for the diagnosis of interventricular septal defect.

Although a clear assessment of each patient is important for predicting the prognosis, in the present analysis, the right atrial pressure was underestimated by transthoracic color Doppler echocardiography compared with right heart catheterization $(\mathrm{P}<0.0001)$. The right atrial pressure is a component of the calculation of the systolic pulmonary artery pressure (11) and mean pulmonary artery pressure (9). Therefore, it is obvious that they were also underestimated by echocardiography $(\mathrm{P}<0.0001$ for both) compared to right heart catheterization. The results of the present study were consistent with those of two prospective studies $(9,15)$, but results of the right atrial pressure were not consistent with those of a retrospective investigation (16) and another prospective study (17). Transthoracic color Doppler echocardiography is routinely used for measurement of the right atrial pressure; the determination of the right atrial pressure is helpful for the prognosis of the ventricular aneurysm and interventricular septal perforation after acute myocardial infarction but not useful for the diagnosis of pulmonary hypertension (18). However, the systolic pulmonary artery pressure is the only parameter that is useful for the diagnosis of pulmonary hypertension (10) and the recommended equation for the mean pulmonary artery pressure was used for calculation (9). Inaccuracy is always a problem in the determination of the right atrial pressure by color Doppler echocardiography (17). Doppler echocardiography is frequently inaccurate in the estimation of pulmonary artery pressure.

Significant differences between the pulmonary capillary wedge pressure determined by right heart catheterization technique and that obtained by transthoracic color Doppler echocardiography modality were obtained in the present study $(\mathrm{P}<0.0001)$. The pulmonary capillary wedge pressure is a strong and accurate measure of left atrial pressure (19). For hemodynamic parameters/pulmonary capillary wedge pressure, transthoracic color Doppler echocardiography has a lower predictive value than right heart catheterization (20). The results of the present study were consistent with those of prospective studies $(9,13)$. It may be concluded that right heart catheterization is essential for the diagnosis of pulmonary capillary wedge pressure.

Of note, the present study had various limitations. For instance, the analysis was retrospective. The patients were in treatment when the diagnosis was performed. However, treatment-naïve patients should be used to obtain meaningful results. Invasive and non-invasive methods were performed simultaneously but the simultaneous measurements of transthoracic color Doppler echocardiography may have been inaccurate due to the suboptimal status of the patients (13). The measurements did not reflect the baseline status of the patients. A delay by $24 \mathrm{~h}$ between invasive and non-invasive methods is acceptable (21) but a delay of $>1$ day was observed between both in a few cases. Correct alignment of the ultrasound beam is crucial for accurate measurements by echocardiography (13). It was not possible to maintain correct alignment of the ultrasound beam for patients in a few cases.

In conclusion, the prediction of the right heart anatomy and physiology by different imaging modalities is crucial besides right heart catheterization in patients with suspected ventricular aneurism with interventricular septum perforation after acute myocardial infarction. Transthoracic color Doppler echocardiography provided information for radiological measurements but underestimated hemodynamic measurements. Further research is required to validate the results of transthoracic color Doppler echocardiography for the evaluation of pulmonary hypertension.

\section{Acknowledgements}

Not applicable.

\section{Funding}

No funding was received.

\section{Availability of data and materials}

The datasets used and/or analyzed during the present study are available from the corresponding author on reasonable request.

\section{Authors' contributions}

All authors read and approved the manuscript for publication. JW contributed to project administration, conceptualization, software and literature review for the study. MY contributed to the formal analysis, validation, resources and literature review of the study. YC contributed to data curation, investigation, resources and literature review. LC contributed to the formal analysis, data curation, resources and literature review. SH contributed to formal analysis and literature review, as well as drafting, review and editing of the manuscript for intellectual content. The authors agree to be accountable for all aspects of the work, ensuring integrity and accuracy.

\section{Ethics approval and consent to participate}

The protocol (no. ZPP/CL/15/2019 dated 4 March 2019) was approved by the review board of People's Hospital of Hangzhou Medical College. An informed consent form was signed by all enrolled patents regarding pathology, anesthesia, radiology and to have an additional procedure(s) purely for research purpose(s) during hospitalization. The study reporting adheres to the STrengthening the Reporting of Observational Studies in Epidemiology statement: A cross-sectional study, the law of China and the Declaration of Helsinki (v. 2008). 


\section{Patient consent for publication}

An informed consent form was signed by all enrolled patents regarding publication of the study, including personal data and images in all forms of citable materials irrespective of time and language during hospitalization.

\section{Competing interests}

Authors declare that they have no competing interests.

\section{References}

1. Marzlin KM: Ventricular aneurysm: Diagnosis and treatment AACN Adv Crit Care 28: 391-394, 2017.

2. Belkhadir M, MoutakiAllah Y, Raissouni Z, Abdou A, Bamous M, Nya F, Atmani N, Houssa MA, El Bekkali Y and Boulahya A: Left ventricular aneurysm and interventricular communication complicating myocardial infarction. Pan $\mathrm{Afr}$ Med J (French) 17: 321, 2014

3. Cho JH, Sattiraju S, Mehta S and Missov E: Delayed ventricular septal rupture complicating acute inferior wall myocardial infarction. BMC Res Notes 6: 124, 2013.

4. Kaluzna-Oleksy M, Araszkiewicz A, Migaj J, Lesiak M and Straburzyńska-Migaj E: 'From right to left': The role of right heart catheterization in the diagnosis and management of left heart diseases. Adv Clin Exp Med 26: 135-141, 2017.

5. Hakuno D, Isobe S, Masaki N and Adachi T: Right ventricular wall dissection with ventricular septal rupture following myocardial infarction visualized on 3-dimensional transthoracic echocardiography. Circ J 79: 2072-2074, 2015.

6. Nagueh SF, Smiseth OA, Dokainish H, Andersen OS Abudiab MM, Schutt RC, Kumar A, Gude E, Sato K, Harb SC and Klein AL: Mean right atrial pressure for estimation of left ventricular filling pressure in patients with normal left ventricular ejection fraction: Invasive and noninvasive validation. J Am Soc Echocardiogr 31: 799-806, 2018.

7. Trivedi KR, Aldebert P, Riberi A, Mancini J, Levy G, Macia JC, Quilicci J, Habib G and Fraisse A: Sequential management of post-myocardial infarction ventricular septal defects. Arch Cardiovasc Dis 108: 321-330, 2015.

8. Shah S, Boyd G, Pyne CT, Bilazarian SD, Piemonte TC, Jeon C and Waxman S: Right heart catheterization using antecubital venous access: Feasibility, safety and adoption rate in a tertiary center. Catheter Cardiovasc Interv 84: 70-74, 2014.

9. Doutreleau S, Canuet M, Enache I, Di Marco P, Lonsdorfer E, Oswald-Mammoser M and Charloux A: Right heart hemodynamics in pulmonary hypertension-An echocardiography and catheterization study. Circ J 80: 2019-2025, 2016.

10. Galie N, Hoeper MM, Humbert M, Torbicki A, Vachiery JL, Barbera JA, Beghetti M, Corris P, Gaine S, Gibbs JS, et al: Guidelines for the diagnosis and treatment of pulmonary hypertension: The task force for the diagnosis and treatment of pulmonary hypertension of the European Society of Cardiology (ESC) and the European Respiratory Society (ERS), endorsed by the International Society of Heart and Lung Transplantation (ISHLT). Eur Heart J 30: 2493-2537, 2009.
11. Rudski LG, Lai WW, Afilalo J, Hua L, Handschumacher MD, Chandrasekaran K, Solomon SD, Louie EK and Schiller NB: Guidelines for the echocardiographic assessment of the right heart in adults: A report from the American Society of Echocardiography endorsed by the European Association of Echocardiography, a registered branch of the European Society of Cardiology, and the Canadian Society of Echocardiography. J Am Soc Echocardiogr 23: 685-713, 2010.

12. Beigel R, Cercek B, Luo H and Siegel RJ: Noninvasive evaluation of right atrial pressure. J Am Soc Echocardiogr 26: 1033-1042, 2013.

13. Kouzu H, Nakatani S, Kyotani S, Kanzaki H, Nakanishi N and Kitakaze M: Noninvasive estimation of pulmonary vascular resistance by Doppler echocardiography in patients with pulmonary arterial hypertension. Am J Cardiol 103: 872-876, 2009.

14. Nand N, Singla SK and Magu S: Cardiac abscess with ventricular aneurysm secondary to old myocardial infarction. J Assoc Physicians India 65: 95-96, 2017.

15. Fisher MR, Forfia PR, Chamera E, Housten-Harris T, Champion HC, Girgis RE, Corretti MC and Hassoun PM: Accuracy of Doppler echocardiography in the hemodynamic assessment of pulmonary hypertension. Am J Respir Crit Care Med 179: 615-1621, 2009.

16. Kasai H,Matsumura A,Sugiura T, Shigeta A, Tanabe N, Yamamoto K, Miwa H, Ema R, Sakao S and Tatsumi K: Mean pulmonary artery pressure using echocardiography in chronic thromboembolic pulmonary hypertension. Circ J 80: 1259-1264, 2016.

17. Aduen JF, Castello R, Daniels JT, Diaz JA, Safford RE, Heckman MG, Crook JE and Burger CD: Accuracy and precision of three echocardiographic methods for estimating mean pulmonary artery pressure. Chest 139: 347-352, 2011.

18. Saggar R and Sitbon O: Hemodynamics in pulmonary arterial hypertension: Current and future perspectives. Am J Cardiol 110 (Suppl): 9S-15S, 2012.

19. Nagy AI, Venkateshvaran A, Dash PK, Barooah B, Merkely B, Winter R and Manouras A: The pulmonary capillary wedge pressure accurately reflects both normal and elevated left atrial pressure. Am Heart J 167: 876-883, 2014.

20. Hoeper MM, Bogaard HJ, Condliffe R, Frantz R, Khanna D, Kurzyna M, Langleben D, Manes A, Satoh T, Torres F, et al: Definitions and diagnosis of pulmonary hypertension. J Am Coll Cardiol 62: D42-D50, 2013.

21. Vlahos AP, Feinstein JA, Schiller NB and Silverman NH: Extension of Doppler-derived echocardiographic measures of pulmonary vascular resistance to patients with moderate or severe pulmonary vascular disease. J Am Soc Echocardiogr 21: 711-714, 2008.

(i) $($ ) This work is licensed under a Creative Commons Attribution-NonCommercial-NoDerivatives 4.0 International (CC BY-NC-ND 4.0) License. 